\title{
2D Mass-spring-like Model for Prediction of a Sponge's Behaviour upon Robotic Interaction
}

\author{
Veronica E. Arriola-Rios and Jeremy Wyatt
}

\begin{abstract}
Deformable objects abound in nature, and future robots must be able to predict how they are going to behave in order to control them. In this paper we present a method capable of learning to predict the behaviour of deformable objects. We use a mass-spring-like model, which we extended to better suit our purposes, and apply it to the concrete scenario of robotic manipulation of an elastic deformable object. We describe a procedure for automatically calibrating the parameters for the model taking images and forces from a real sponge as ground truth. We use this ground truth to provide error measures that drive an evolutionary process that searches the parameter space of the model. The resulting calibrated model can make good predictions for 200 frames ( 6.667 seconds of real time video) even when tested with forces being applied in different positions to those trained.
\end{abstract}

\section{Introduction}

The objective of this research is the study of the process of physical modelling, prediction and evaluation of the predictive capabilities of a mass-spring-like model (as a prerequisite for planning), applied to the concrete scenario of robotic manipulation of an elastic deformable object (dish washing sponge). The robot identifies a region of the world, where its force sensor detects opposition to movement, and whose shape and behaviour can be modelled. This region can be better observed though a colour camera. A simple colour segmentation algorithm allows for the identification and tracking of the region's behaviour. A regular triangular mesh has been chosen as

Veronica E. Arriola-Rios

University of Birmingham, Edgbaston, Birmingham, B15 2TT, U.K. e-mail: veronica.esther@gmail.com

Jeremy Wyatt

University of Birmingham, Edgbaston, Birmingham, B15 2TT, U.K. e-mail: jlw@cs.bham.ac.uk 
the form of representation of this region, so that the mass-spring-like model can be applied to it. A search for the best set of parameters for the model is conducted by evaluating the similarity of the behaviour of the modelled sponge vs. the real sponge in the 2D sequence of images. The generalisability of the resulting set is tested on data gathered when the forces were applied on other parts of the sponge.

The aim of the robot is to find a way to calibrate a model, so that it can describe the behaviour of an occupied physical region (sponge), just with the help of the information it can receive from its sensors (a camera and a force sensor) during a few interactions with it (pushing it against an obstacle), and a set of basic knowledge of how to learn to calibrate those models (search algorithms). From here, the set of requirements for the representation of the deformable object are explained in Sect. 3.

For the simulation, we opted for what seemed to be a simple general physics based model proposed by Teschner[22]. However, in order to better reproduce the observed behaviours, some modifications and extensions where introduced, like a new force term that tends to preserve the angles between the springs in the mesh, as it is explained in Sect. 4.

Finally, Sect 5 presents the set of experiments where a Katana arm [14], equipped with a rigid finger, pushed a sponge against an obstacle (a pencil, fixed and perpendicular to the plane of the sponge). See Fig. 1. Given a good set of parameters, the program can take as inputs the position of the obstacles for every frame (finger and pencil), the initial position and shape of the sponge, and the sensed force in the direction of movement for every frame, and it will be able to predict the deformation of the sponge for the remaining frames in the video (200 frames, 6.667 seconds) with very good accuracy. Such good sets can be found though a systematic search of the space of parameters or by a simple genetic algorithm. Sect 6 summarises our conclusions.

\section{Related Work}

It is hard to catalogue the literature about deformable objects, because it covers a wide range of aspects which can be combined to obtain good simulations for different situations (we need to start as general as possible, but without loosing the other ones from sight), starting with computer vision (which covers: identification, representation [17, 15], classification, tracking [9]), simulation and manipulation (with applications mainly in robotics, medicine [12], computer graphics [16, 7] and industry [19]). Sometimes the technique involves manually characterising the behaviour of a family of materials [18], sometimes the main focus is in topological information [20], sometimes they overlap across fields or get combined for new applications, like the work by Luo and Nelson [10] where visual tracking can provide haptic information, after a calibration phase of a FEM model links vision and haptic feedback. The first aim of Computer Graphics is to provide with rich, general and flexible representations of deformable objects: meshes and splines cover these requirements in different ways [21], different behaviours can be attained by applying 


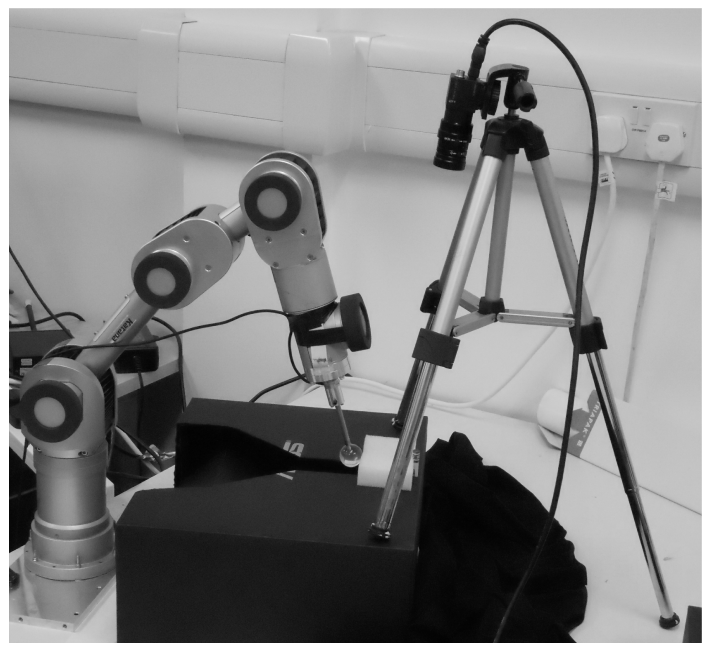

Fig. 1 Experimental setup. Viewing from a side, a one fingered Katana arm with a force sensor pushes a sponge. The transparent finger pushes the sponge away from it. On the other end, a pencil is used as an obstacle always opposed to the finger. A camera observes and records the action from the top.

transformations to vertices and control points, respectively, or by calculating its new possition individually, as it is done in this work.

Physics based models are commonly used either to reinforce tracking or to produce simulations. Two families of them pervade the scientific literature for deformable objects [4]:

Finite Element Methods (FEM): Objects are divided into unitary surface or volumetric elements joined at discrete node points where a continuous equilibrium equation is approximated over each element. To solve the system, large matrices connecting the elements must be solved. There are several variations of these: Non-linear FEM, Geometric Nonlinear FEM and Boundary Element Methods (which transforms the equations so that they have to be integrated at the border of the object instead of the whole volume). The Long Elements Method uses three mutually perpendicular reference planes that cross the object, and the relative positions of points inside the object with respect to these reference planes are simulated [1].

Mass-spring Methods: Objects are represented by meshes where masses are located at the nodes and the edges correspond to springs connecting them. It is possible to discretise and integrate the system of equations for each mass point separately, making the process easy and parallelisable. It can be easily applied to model volumes or $2 \mathrm{D}$ textiles [6].

In the area of predictive tracking, the work by Malassiotis [11] resembles the structure of our approach (even though the underlying model is different): a triangular mesh is calculated to cover the 2-D image of a textured deformable object of 
interest. The aim of his technique is to identify the principal deformation modes in the mesh over the observed object, so that future deformations can be expressed as combinations of these modes. The 2-D shape of the object is modelled with an elastic membrane deformed by virtual forces, its strain energy is calculated by making use of the finite element theory, which causes it to be costly in time and complexity. This information will guide the deformations of the mesh, while trying to predict how the object will be deformed. However, the shapes it can describe are limited by what can be represented as combinations of the selected modes.

Among the most innovative works with mass-spring methods are: Burion et al. [3], who use a mass-spring skeleton model [4], where filling spheres are placed along the medial skeleton of the object and are connected together with elastic links, which model elongation, flexion and torsion properties. They use a particle filter algorithm to estimate the stiffness coefficients. Since we plan to extend our work to plasticine, the requirement of a skeleton make this approach unsuitable for our purposes.

Nevertheless, we chose to work with Teschner's model [22], following the experience of Morris [13] (in computer graphics), because it addresses the same issues but is easily implemented in 2D (the dimensionality of our ground truth). Here, additionally to the traditional damped springs at the edges, two new energy terms are devised to enforce preservation of area and volume, making this model more adequate to simulate a broad range of realistic behaviours. Also, the terms for distance, area and volume preservation have the same structure, this makes the model uniform and straightforward to implement and extend. Particularly, we added a term to consider preservation of angles, which considerably improved the stability of the simulation.

Also Barbara Frank works with learning of deformable objects, in colaboration with Teschner. Their work has the same general structure than ours, but they use a 3D FEM model for the simulation and search for calibration parameters using a gradent descent algorithm[5].

\section{Representation of the Deformable Object}

It is clear that it took centuries for humans to discover the molecular and atomic structure of matter, some time more to understand its interactions, how to control the creation of particular structures, and how the macroscopic attributes emerge from the underlying microscopic composition of the material [8]. Nevertheless, there was an intelligent process that allowed humans to handle materials even before they had this knowledge. Since in this work we are interested in the programming of cognitive robots, the model to be used does not have to be physically correct, but its behaviour must correspond with the observations, and it must be possible to apply it easily to a wide range of materials and shapes.

There is a set of simple requirements, given by the predictive task we have in mind: 
1. The representation must be deformable, just as the original material.

2. Given that we are receiving visual information in $2 \mathrm{D}$, we will try to keep things simple by having an internal representation also in $2 \mathrm{D}$.

3. It must be possible to represent interactions between solids (rigid and deformable). Particularly, we need to detect contact.

4. The model must provide information beyond the points where data was collected [interpolation and extrapolation] e.g. beyond the points where testing forces were applied and deformations or displacements were registered. Observe that, in particular, the force sensor in our experiment only provides readings in one point, but the shape of the robotic finger is actually a sphere. Also, the initial shape and position of the mesh is given for the first frame, but the model must be able to deform the initial representation accordingly.

A very common representation of any object in computer graphics is a mesh. A mesh can be easily rendered, transformed (translated, rotated, resized, etc.) and deformed. It is also quite common to use meshes for physics based methods. Each node has a mass, and neighbour nodes are connected by edges. Since we are interested in real time modelling of dynamic behaviour, the evaluation of a mass-spring model is appealing, therefore, the mesh must be designed taking this into account. For mass-spring models, the shape of the mesh can lead to undesired anisotropic behaviours, since forces are applied only through the edges (which become springs) [2]. To attend this, to a certain extent, we opted for taking the simple approach of generating a symmetric triangular mesh.

\section{Physics Model}

\subsection{Antecedents}

To plan complex motions in AI a planner may find paths between keyframes and displacements of keypoints. It is thus an important task for learning algorithms to find these keypoints. In the case of deformable objects, the new shapes an object can take may be characterised by keypoints like points of high curvature. Nevertheless, they are still so varied that it is not possible to detect and remember them all in advance. Therefore, an algorithm that can predict the continuous or discontinuous transformations of key points in the deformation can help to explore the possible consequences of plans that include nove interactions whose key points are still unknown. In order to do that, the underlying representation of the model must not depend on those unknown keypoints. That is why a regular mesh has been proposed as the basis for the model we use. In future work, this mesh could be used to extract the keypoints mentioned.

This research approach is inspired by the work by Morris[13]. He works with a 3D finite element model as a ground truth to automatically calibrate an almost equivalent instance of the mass-spring model proposed by Teschner [22], which can 
be used instead for real time simulations. Random sets of parameters are proposed and evaluated at equilibrium positions, an adaptive simulated annealing search is used to modify the best parameters of the springs and look for better candidates. Observe that only the final equilibrium positions are evaluated, the forces are applied on vertices of the mesh and the position of the objects is fixed.

\subsection{Inputs}

The katana arm has been equipped with a 6 degrees sensor (forces and torques), but for the experiments in this research only one direction is relevant. A colour camera with a resolution of $800 \times 600$ pixels is placed perpendicular to the direction of movement so that it can capture the movement of the finger and the deformation of the sponge. See Fig. 2.

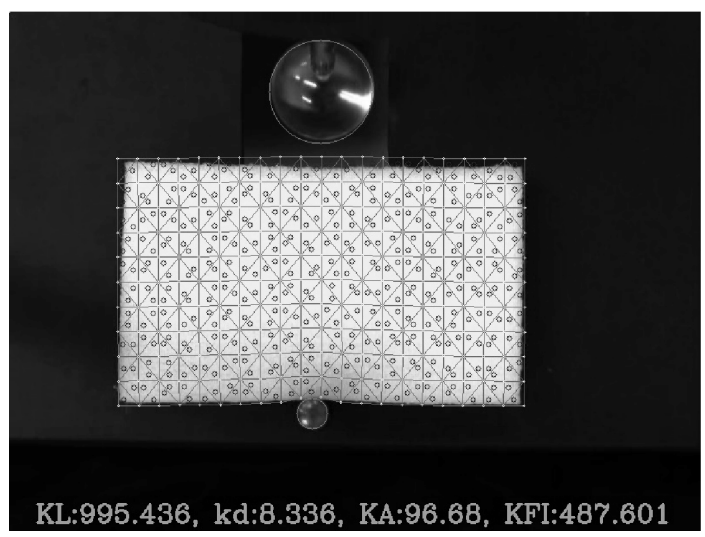

Fig. 2 Viewing from the top, a one fingered Katana arm with a force sensor pushes a sponge. The transparent finger pushes the sponge downwards. On the other end (below), a pencil (cap) is used as an obstacle always opposed to the finger. Both obstacles are represented by thin circles in the photo.

\subsection{Graphical Constraints}

Given that the main focus of this work is on the deformable object, the treatment of the rigid objects involved has been simplified as much as possible. Both, the finger and the pencil are represented as instances of hard circular obstacles. This implies that any element of the deformable mesh will not be allowed to enter or cross over the enclosed region. This constraint is enforced at every frame during the simulation through a standard set of collision detection subroutines. Also the 
triangulation must remain without crossings. Whenever the displacemente of a node produces a crossing, the opposite ending of the affected triangle and the node itself get pushed in opposite directions to undo the error. If a set of parameters can not respect this constraints it is eliminated.

\subsection{From Energy Constraints to Force terms}

The core of the model is in the energy terms that enforce the preservation of three quantities: length of the springs, area of each triangle and the internal angles of the triangles. The first two terms are directly derived for the two dimensional case following Teschner's method and can be seen in [22]. The last term is our original contribution.

Equal masses are allocated at the nodes of the mesh and the edges correspond to the springs. The dynamics of the deformation of the objects are represented through dynamic constraints from which potential energies are obtained. The dynamics of the system are ruled by the forces that minimise these energies. Teschner indicates how to derive those forces and Morris [13] uses geometrical arguments to explain them. The following sections indicate the corresponding expressions.

\subsubsection{Preservation of Distance:}

The spring will tend to recover its original length. Strictly this is the only term that will force the triangles to recover their original shape. See Fig. 3.a.

Force:

$$
F_{D}\left(p_{i}\right)=k_{D}\left(\left|p_{j}-p_{i}\right|-D_{0}\right)\left(\frac{p_{j}-p_{i}}{\left|p_{j}-p_{i}\right|}\right)
$$

Where $p_{i}, p_{j}$ are the mass point positions, $k_{D}$ the stiffness coefficient, $E_{D}$ the potential energy based on the difference between the current distance of two points and the initial or rest distance $D_{0}$, with $D_{0} \neq 0 . F_{D}$ is the force resulting from this energy and it will pull or push the masses in the direction of the line that joins them.

\subsubsection{Preservation of Surface Area:}

Every triangle in the mesh tries to recover its area. This term does not respect the original shape of the triangle, thus allowing the hole mesh to find a new equilibrium even if greatly deformed. See Fig. 3.b. 
Force:

$$
\begin{aligned}
F_{A}\left(p_{i}\right) & =k_{A} \cdot \text { forcemag }_{A}\left(p_{i}\right) \cdot \text { forcedir }_{A}\left(p_{i}\right) \\
\operatorname{forcemag}_{A}\left(p_{i}\right) & =\frac{\frac{1}{2}\left(\left(p_{k}-p_{i}\right) \times\left(p_{j}-p_{i}\right)\right)-A_{0}}{A_{0}} \\
\operatorname{forcedir}_{A}\left(p_{i}\right) & =\frac{F_{A}\left(p_{i}\right)}{\left|F_{A}\left(p_{i}\right)\right|}=\frac{\text { areagradient }\left(p_{i}\right)}{\mid \text { areagradient }\left(p_{i}\right) \mid} \\
\operatorname{areagradient}\left(p_{i}\right) & =\left(p_{i}-p_{j}\right)-\left(\left(p_{k}-p_{j}\right) \cdot \frac{\left(p_{k}-p_{j}\right) \cdot\left(p_{i}-p_{j}\right)}{\left(p_{k}-p_{j}\right) \cdot\left(p_{k}-p_{j}\right)}\right)
\end{aligned}
$$

Where the energy $E_{A}$ considers triples of mass points that build surface triangles. $E_{A}$ represents energy based on the difference of the current area of a surface triangle and its initial area $A_{0}$ with $A_{0} \neq 0$. Here $k_{A}$ is an area stiffness coefficient. Each mass with move in the direction of the height of the triangle that passes through it, to increase or decrease its area.

a.

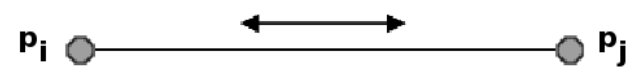

b.

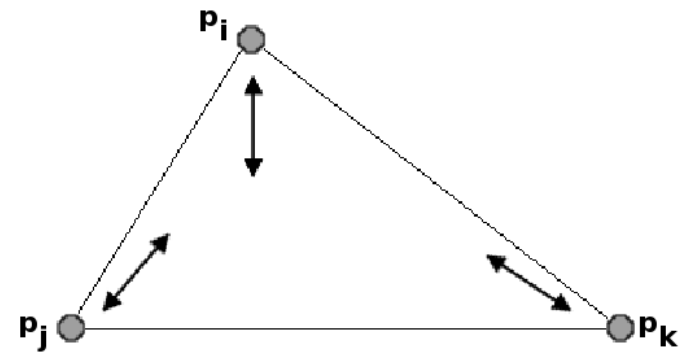

Fig. 3 a. The linear force of the spring pulls inside if the spring was elongated, or outside if it was compressed. $b$. The triangle tries to quickly recover its area by pulling all its vertices along its corresponding heights.

\subsubsection{Preservation of Angles:}

We added an extra term to enforce the preservation of angles. The energy depends on the difference of the angles between adjacent edges. The force emerging from this term is harder to visualize, it is a linear combinations of the vectors along the edges that form the angle of interest, it pretends to restore the original angle, but does not pay attention to the original size. See Fig. 4. Therefore, it helps recover a 
similar triangle, but if used alone can collapse or explode the triangle. An additional line in the code also forces an inverted angle to recover its original orientation.

Energy:

$$
\begin{aligned}
E_{\varphi}(\varphi) & =\frac{1}{2} k_{\varphi}\left(\varphi-\varphi_{0}\right) * \\
\varphi\left(p_{i}, p_{j}, p_{k}\right) & =\arccos \left(\frac{\left(p_{j}-p_{i}\right) \cdot\left(p_{k}-p_{i}\right)}{\left\|p_{j}-p_{i}\right\|\left\|p_{k}-p_{i}\right\|}\right)^{2}
\end{aligned}
$$

* It was also considered to multiply $E_{\varphi}$ by the lengths of the edges, but it hasn't improved the performance of the model.

Where $\varphi$ is the angle between adjacent edges, $E_{\varphi}$ is the energy associated to changes in the angle and $k_{\varphi}$, the corresponding stiffness constant. Contrary to the previous cases, it is not so evident in which direction the force will act.

Force:

$$
\begin{aligned}
F_{\varphi}\left(p_{i}\right) & =k_{\varphi}\left(\varphi-\varphi_{0}\right) \frac{\partial \varphi}{\partial p_{i}} \\
\frac{\partial \varphi}{\partial p_{i}}\left(p_{i}\right) & =\frac{1}{d_{j i} d_{k i} \sqrt{1-\left[\frac{p p}{\left(d_{j i}\right)\left(d_{k i}\right)}\right]^{2}}}\left\{\left[1-\frac{p p}{d_{k i}^{2}}\right]\left(p_{k}-p_{i}\right)+\left[1-\frac{p p}{d_{j i}^{2}}\right]\left(p_{j}-p_{i}\right)\right\} \\
p p\left(p_{i}\right) & =\left(p_{j}-p_{i}\right) \cdot\left(p_{k}-p_{i}\right) \\
d_{j i}\left(p_{i}\right) & =\left\|p_{j}-p_{i}\right\| \\
d_{k i}\left(p_{i}\right) & =\left\|p_{k}-p_{i}\right\|
\end{aligned}
$$

The differential equations that rule the behaviour of the system are integrated with a numerical approach. Originally the acceleration of the masses is proportional to the force, the equation must be integrated twice to obtain the positions as a function of time. In this case, in order to compute the new state of the system $(x(t+h)$ position at time $t+h, v(t+h)$ velocity at time $t+h)$ the Verlet integration scheme is used [6].

$$
\begin{gathered}
x(t+h)=2 x(t)-x(t-h)+h^{2} \frac{\mathbf{F}(\mathbf{t})}{m}+O\left(h^{4}\right) \\
v(t+h)=\frac{x(t+h)-x(t-h)}{2 h}+O\left(h^{2}\right)
\end{gathered}
$$

with $F(t)=F_{D}(t)+F_{A}(t)+F_{\varphi}(t)$, and $m$, the mass of the node. 
c.

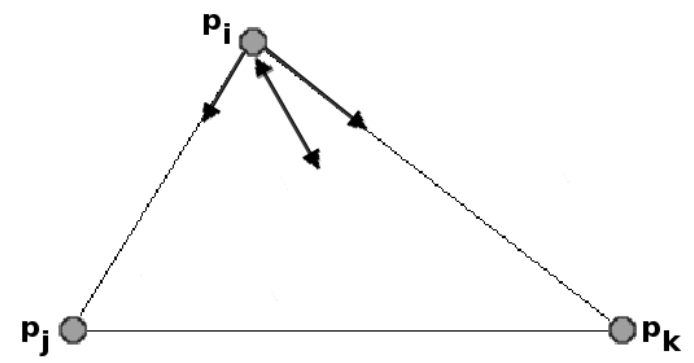

Fig. 4 The angular force displaces the vertex of interest in the direction of maximum change of the angle in order to recover its original value. This direction is a linear combination of the vectors that emerge from both edges forming the angle, and does not respect the original size of the triangle.

This will yield the typical oscillatory behaviour of springs. However, to simulate a heavily damped sponge we made the velocities proportional to the forces. Now, instead of having a second order set of equations, the set is first order. The simulations we obtained were closer to the observed behaviour. Also, for every frame in the simulation, there are 10 steps in the numerical integration.

$$
x(t+h)=x(t)+h \frac{\mathbf{F}(\mathbf{t})}{m}
$$

with $F(t)=F_{D}(t)+F_{A}(t)+F_{\varphi}(t)$.

However, to give stability to the numerical integration process it is necessary to add damping terms to the linear force. Teschner gives the following formula to calculate the force:

$$
F^{i}\left(p_{0}, \ldots, p_{n-1}, v_{0}, \ldots, v_{n-1}\right)=\left(-k C-k_{d} \sum_{0 \leq j<n} \frac{\partial C}{\partial p_{j}} v_{j}\right) \frac{\partial C}{\partial p_{i}}
$$

Given that $F_{D}$ for every $p_{i}$ only depends on $p_{i}$ and $p_{j}$, the sum has two terms.

$$
F_{D}\left(p_{i}, p_{j}\right)=\left(k_{D}\left(\frac{\left|p_{j}-p_{i}\right|-D_{0}}{D_{0}}\right)+k_{d} \frac{1}{D_{0}} \frac{p_{j}-p_{i}}{\left|p_{j}-p_{i}\right|} \cdot\left(v_{j}-v_{i}\right)\right) \frac{1}{D_{0}}\left(\frac{p_{j}-p_{i}}{\left|p_{j}-p_{i}\right|}\right)
$$

Still these forces only represent the internal tension of the material that makes it tend to recover its shape. The external force applied by the finger and measured by the sensor is added to the forces acting on the nearest vertex to the finger in the mesh. Additionally, the mesh must respect the graphical constraint imposed by the finger, which greatly helps in shaping the sponge, and propagates the effect caused by the hole circular shape.

At the beginning we assumed that the spring terms would suffice even to reproduce the slight translation of the sponge as it is pushed by the finger, since pushing a spring should make this push its neighbours and so on. However, our first experi- 
ments showed it not to be the case. The effect of pushing a spring get diluted among the deformation of the spring and the area and angular terms. In order to obtain the translation, we must add a solid propagation of the finger's force. This is, that part of the force is absorbed in deformation, while another amount affects all vertices and produces an even displacement. For the moment we just added an extra parameter to the model that fixes the amount of force that is invested in translation. There is an additional parameter $f_{t}$ that represents a solid force that is constantly propagated to the entire sponge (to every vertex) and accounts for the overal solid translation of the object.

The algorithms where implemented in $\mathrm{C}++$, making use of the GNU compiler gcc 4.4.4. The triangulations where managed with the CGAL library, and the vision part was handled with OpenCV.

\section{Experiments}

\subsection{Evaluation: Difference of Areas}

The function used to automate the decision of what makes a good set of parameters is quite simple, but provides a sufficient criterion to eliminate bad sets of parameters. It consists in measuring the difference between the area occupied by the image of the sponge and the area covered by the mesh of the model (this is done pixel by pixel). The bigger this difference, the worst the model. All the differences frame by frame are added up to assign a mark to the set of parameters during the hole duration of the video. See Fig. 5.

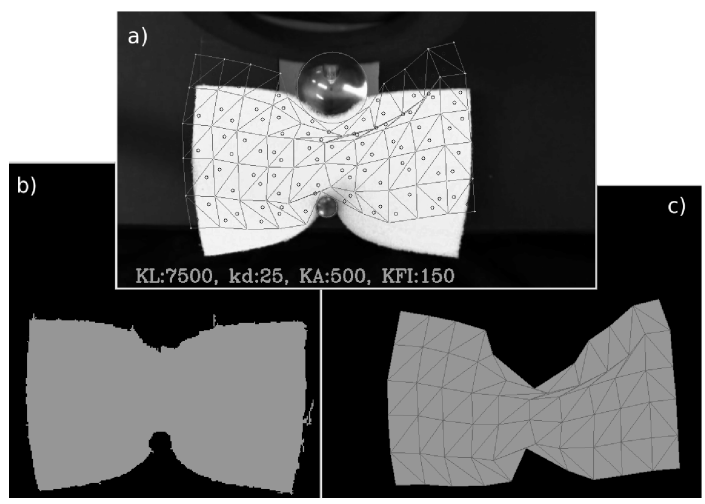

Fig. 5 a) Shows the original image with the mesh overlaid as the simulation is executed. b) The area occupied by the sponge. c) The area occupied by the mesh. The evaluation function counts the pixels in the difference: those in the sponge that are not in the mesh, and those in the mesh that are not in the sponge. The positions are absolute. 


\subsection{Results}

The numerical value of the forces measured in the relevant direction of movement range from $-3.8 \mathrm{~N}$ (due to noise in the sensor) to $170.3 \mathrm{~N}$. We use a simple genetic algorithm to find good sets of parameters. Here, the first generation has only random values for every parameter, within the ranges: $k_{D} \in[0.001,1000.0]$, $k_{d} \in[0.001,100.0], k_{A} \in[0.001,200.0], k_{\varphi} \in[0.001,700.0]$ and $f_{t} \in[0.0,200.0]$. After, for every generation, one third of the new elements are the best of the previous generation (they don't get evaluated again), one third are the previous ones plus Gaussian noise (the value of sigma gets reduced for every generation from 100 to $10)$, and the last third are new random elements. With the genetic algorithm, after the 8th generation the set of parameters with the best mark has been the same. It produced the videos summarized in Fig. 6, and the marks for the best sets are summarized in Table 1. However, other executions of the same algorithm have found other good sets of values (any of them would serve the purposes of the robot). Nevertheless we completed the analysis with a systematic run.

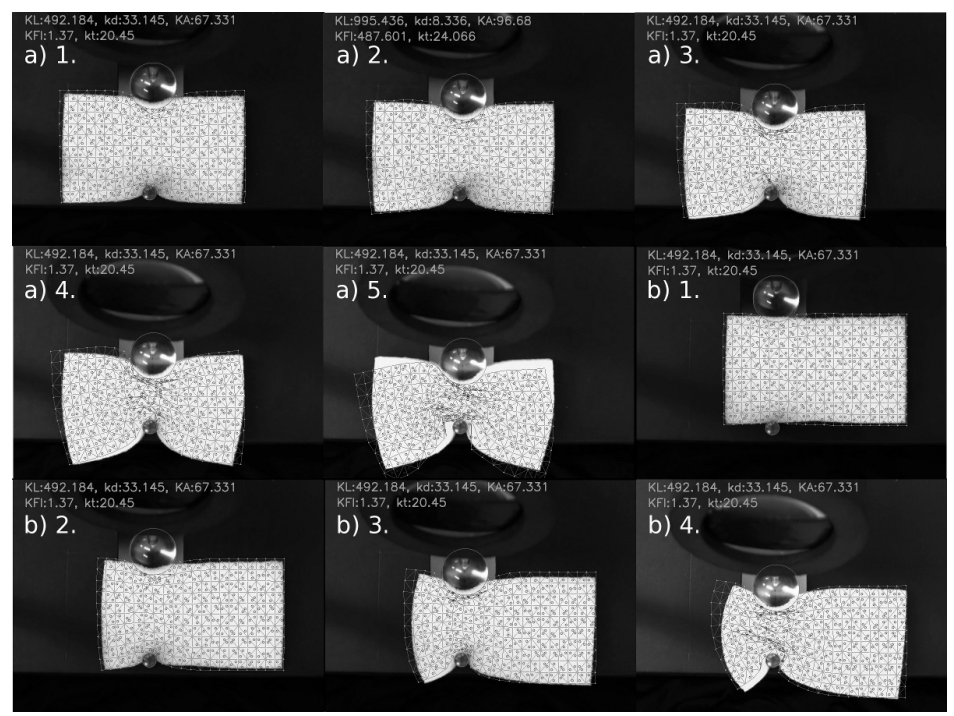

Fig. 6 Sequence taken from a simulation with the best set as evaluated by a genetic algorithm: $k_{D}=492.184, k_{d}=33.145, k_{A}=67.331, k_{\varphi}=1.37$ and $f_{t}=20.45$. a) Over the video used for the evaluation of the parameters. a) 5 . In the last frame an error remains due to the oversimplification of the translation function: the elements of the sponge keep being pushed down even after the finger has stopped moving (frame 300). b) Same model and parameters but with the forces acting on different positions. The predictions are fairly correct for 200 frames. We have another set where the forces are applied to the right side of the sponge, the results are symmetric, but are not shown for lack of space.

We noticed that big values for the preservation of areas create severe problems while trying to maintain the graphical constraints (T-test $t=8.33$, for all the follow- 
2D Mass-spring-like Model for Prediction

Table 1 Best sets of parameters for every generation.

\begin{tabular}{lllllll}
\hline Generation & Mark & $k_{D}$ & $k_{d}$ & $k_{A}$ & $k_{\varphi}$ & $f_{t}$ \\
\hline 0 & $53,656,529$ & 96.501 & 68.205 & 112.012 & 572.616 & 17.3 \\
1 & $44,993,321$ & 613.188 & 13.7061 & 85.1941 & 168.793 & 18.6731 \\
3 & $42,767,941$ & 249.164 & 23.855 & 145.933 & 276.542 & 19.326 \\
8 & $41,291,354$ & 492.184 & 33.145 & 67.331 & 1.37 & 20.45 \\
\hline
\end{tabular}

For the generations not shown the values were repeated

ing: $d f=3274, p<0.002$ ), large values of $k_{\varphi}$ help (T-test $t=9.32$ ) and $f t=15$ is the best tested value (T-test $t=246.6$ ). 53.5\% of the sets of parameters in the range of values we considered $\left(k_{D} \in[600.0,1000.0], k_{d} \in[50.0,100.0], k_{A} \in[50.0,200.0]\right.$, $k_{\varphi} \in[50.0,700.0]$ and $\left.f_{t} \in[25.0,200.0]\right)$ got discarded. The best nine results have $k_{D}=1000, k_{d}=50, k_{A} \leq 50, f_{t}=15$ and $k_{\varphi} \in[600.0,700.0]$, with their marks around 52 million. There are also differences in magnitude depending on the number of elements in the mesh. Notice that the genetic algorithm was able to try values in a wider range, and in fact quickly found its best sets outside from what we had considered for the systematic search. If there are more elements in the mesh, the stiffness coefficients are smaller. Unfortunately the systematic search can take up to several weeks in a PC with an Intel(R) Core(TM)2 Quad CPU Q6600 @ 2.40GHz.

\section{Conclusions}

We managed to automatically calibrate a mass-spring-like model taking images and forces from a real sponge as ground truth. The discretisation of the equations of movement produce jumps whose effects are hard to control. The calibrated model works well until opposing forces make it difficult to respect all the graphical constraints. The simulation also fails at the end, when the translation term keeps acting even when the sponge is not moving any more (this requires a better function for the translation component). Furthermore, in order to consider new behaviours (plasticity, rotations, etc.) it is necessary to add new terms to the model and the search space is increased. This shows that, if we want to program a cognitive robot using physical models, it would be required to provide the robot with a big collection of components to consider, which leaves open the question of whether there is a simpler mechanism that can cover many behaviours on the same basis.

Acknowledgements Thank you to Dr. Helge Ritter and Matthias Schoepfer from the University of Bielefeld, Germany for their suggestions leading to the completion of this work. To Rustam Stolkin for his aid to make the experiments and Marek Kopicki for his software to control the arm. Also to Aaron Sloman for interesting discussions and suggestions for this research. To the CONACYT in Mexico and to the ORSAS scheme in the UK for sponsoring my PhD, also the research leading to these results has received funding from the European Community's Seventh Framework Programme [FP7/2007-2013] under grant agreement No. 215181, CogX. 


\section{References}

1. Balaniuk, R., Salisbury, K.: Dynamic simulation of deformable objects using the long elements method. 10th Symposium On Haptic Interfaces For Virtual Environment And Teleoperator Systems, Proceedings pp. 58-65 (2002)

2. Bourguignon, D., Cani, M.P.: Controlling anisotropy in mass-spring systems. In: N. Magnenat-Thalmann, D. Thalmann, B. Arnaldi (eds.) 11th Eurographics Workshop on Computer Animation and Simulation, EGCAS 2000, August, 2000, Springer Computer Science, pp. 113-123. Springer-Verlag, Interlaken, Suisse (2000)

3. Burion, S., Conti, F., Petrovskaya, A., Baur, C., Khatib, O.: Identifying physical properties of deformable objects by using particle filters. 2008 Ieee International Conference On Robotics And Automation, Vols 1-9 pp. 1112-1117 (2008)

4. Conti, F., Khatib, O., Baur, C.: Interactive rendering of deformable objects based on a filling sphere modeling approach. In: Robotics and Automation, 2003. Proceedings. ICRA '03. IEEE International Conference on, vol. 3, pp. 3716 - 3721 (2003)

5. Frank, B., Schmedding, R., Stachniss, C., Teschner, M., Burgard, W.: Learning the elasticity parameters of deformable objects with a manipulation robot. In: Proc. of the IEEE/RSJ International Conference on Intelligent Robots and Systems (IROS) (2010)

6. Fuhrmann, A., Gro, C., Luckas, V.: Interactive animation of cloth including self collision detection. Journal of WSCG 11(1), 141-148 (2003)

7. Gibson, S.F.F., Mirtich, B.: A survey of deformable modeling in computer graphics. Tech. rep., MERL (Mitsubishi Electric Research Laboratory) (1997)

8. Gonzlez-Vias, W., Mancini, H.L.: An Introduction To Materials Science. Princeton University Press, U.S.A. (2004). Translation of: Ciencia de los Materiales

9. Kass, M., Witkin, A., Terzopuolos, D.: Snakes: Active contour models. International Journal Of Computer Vision 1(4), 321-331 (1988)

10. Luo, Y.H., Nelson, B.J.: Fusing force and vision feedback for manipulating deformable objects. Journal Of Robotic Systems 18(3), 103-117 (2001)

11. Malassiotis, S., Strintzis, M.G.: Tracking textured deformable objects using a finite-element mesh. Ieee Transactions On Circuits And Systems For Video Technology 8(6), 756-774 (1998)

12. McInerney, T., Terzopoulos, D.: Deformable models in medical image analysis: a survey. Med Image Anal 1(2), 91-108 (1996)

13. Morris, D., Salisbury, K.: Automatic preparation, calibration, and simulation of deformable objects. Computer Methods In Biomechanics And Biomedical Engineering 11(3), 263-279 (2008)

14. Neuronics: Katana user manual and technical description. http://www.neuronics.ch (2004)

15. Newcombe, R.A., Davison, A.J.: Live dense reconstruction with a single moving camera. In: CVPR (2010)

16. O’Brien, J.F., Bargteil, A.W., Hodgins, J.K.: Graphical modeling and animation of ductile fracture. ACM Trans. Graph. 21(3), 291-294 (2002)

17. Ravishankar, S., Jain, A., Mittal, A.: Multi-stage contour based detection of deformable objects. Computer Vision - Eccv 2008, Pt I, Proceedings 5302, 483-496 (2008)

18. Remde, A., Abegg, F., Worn, H.: Ein allgemainer ansatz zur montage deformierarbarer linearer objekte mit industrierobotern (a general approach for the assembly of deformable linear objects with industrial robots). In: Robotik'2000. Berlin, Germany (2000)

19. Saadat, M., Nan, P.: Industrial applications of automatic manipulation of flexible materials. Industrial Robot 29(5), 434-442 (2002)

20. Saha, M., Isto, P.: Manipulation planning for deformable linear objects. Ieee Transactions On Robotics 23(6), 1141-1150 (2007)

21. Song, Y., Bai, L.: 3d modeling for deformable objects. Articulated Motion And Deformable Objects, Proceedings 5098, 175-187 (2008)

22. Teschner, M., Heidelberg, B., Muller, M., Gross, M.: A versatile and robust model for geometrically complex deformable solids. In: Proceedings of Computer Graphics International (CGI’04), pp. 312-319. Crete, Greece (2004) 